with hypertension (OR 0.900), hyperlipidaemia (OR 0.836) and type 2 diabetes (OR 0.860 ) had significantly lower mortality rates. Adjusted mortality rates were also lower in widowed patients with hypertension (OR 0.973) and in both widowed and separated patients with type 2 diabetes (OR 0.965 and 0.974 , respectively). Conversely, unmarried patients had significantly raised adjusted mortality rates in type diabetes (OR 1.046) and in hypertension (1.034).

Conclusion Single patients with modifiable risk factors have significantly higher mortality rates compared to married patients which could help to explain the raised mortality rates documented in single patients following an acute coronary syndrome. Protective effects of marriage likely result from increased social support leading to healthier lifestyles and increased medication compliance. Our findings suggest a need for increased social support for single patients with modifiable cardiovascular risk factors.

\section{OXIDISED LDL LEVELS CORRELATE WITH LIPOPROTEIN (A) LEVELS AND ARE REDUCED BY LIPOPROTEIN APHERESIS IN A RANDOMISED STUDY ON PATIENTS WITH REFRACTORY ANGINA AND RAISED LIPOPROTEIN(A)}

${ }^{1}$ Tina Khan*, ${ }^{2}$ Dorian Haskard, ${ }^{2}$ Mikhail Caga-Anan, ${ }^{3}$ Dudley Pennell, ${ }^{4}$ Mahmoud Barbir, ${ }^{2}$ Ramzi Khamis. 'Imperial College, Royal Brompton and Harefield NHS Foundation Trust; ${ }^{2}$ Imperial College; ${ }^{3}$ Imperial College, Royal Brompton Hospital; ${ }^{4}$ Royal Brompton and Harefield NHS Foundation Trust

\subsection{6/heartjnl-2017-311726.93}

Introduction Lipoprotein apheresis (LA), an extra-corporeal lipid lowering technique is a promising new therapeutic technique for the treatment of coronary artery disease. Patients with high levels of Lipoprotein(a) but normal cholesterol levels are challenging to treat, and therefore this technique offers a novel therapeutic avenue. Levels of oxLDL are well known to correlate with both the degree of atherosclerosis in patients, as well as their risk of cardiovascular events. Antibodies against oxLDL are recognised to form immune complexes and are implicated in the clearance of oxLDL.

Methods We tested the effect of LA on levels of oxLDL as well and anti-oxLDL antibody levels as a sub-study of a randomised controlled trial in 20 patients with refractory angina, raised $\mathrm{Lp}(\mathrm{a})>500 \mathrm{mg} / \mathrm{L}$ and $\mathrm{LDL}$ cholesterol $<4 \mathrm{mmol} / \mathrm{L}$ (average baseline level was $2.16 \mathrm{mmol} / \mathrm{L}$ ) with three months of blinded weekly LA or sham, followed by crossover. Enzymelinked immunosorbent assays (ELISA) were used to measure levels of MDA-LDL (oxLDL), as well as IgG and IgM anti oxLDL antibody levels at baseline as well as after 3 month periods of active and sham treatment.

Results Baseline oxLDL levels were correlated with both baseline LDL $(r=0.69, p=0.001)$ and $\mathrm{Lp}(\mathrm{a})$ levels $(r=0.63$; $\mathrm{p}=0.03)$. OxLDL was reduced by $30 \%$ after apheresis. There was a mean drop of -0.11 units (U) $(95 \%$ CI $-0.13,-0.09$ ) from $0.37 \pm 0.06$ to $0.26 \pm 0.04$. During sham oxLDL levels did not change significantly with a mean change of $-0.01(95 \%$ CI $-0.04,0.02)$ from $0.35 \pm 0.07$ to $0.34 \pm 0.07 \quad(p<0.0001$ between treatment arms). IgG and IgM anti-oxLDL levels were both reduced by $22 \%$ after apheresis; IgG from 0.61 \pm 0.21 to $0.47 \pm 0.20 ; \quad \mathrm{p}=0.0036$ (optical density units, [ODU]); whilst IgM was reduced by a median value of 0.15 ODU from $0.66 \pm 0.43$ to $0.54 \pm 0.36 ; p=0.012$. IgG and $\operatorname{IgM}$ anti-oxLDL levels were not affected by the sham procedure.
Conclusions LA significantly reduced oxLDL levels as well as anti-oxLDL antibody levels in patients with raised $\mathrm{Lp}(\mathrm{a})$. Whilst the mechanism could be due to the direct binding of all the studied proteins to the charged dextran column of the apheresis apparatus, it is more likely that the reduction of anti-oxLDL antibodies is the result of IgG- and IgM-oxLDL immune complex depletion via lipoprotein extraction by the column. We have previously reported a therapeutic benefit of LA on this patient population. Given the pathogenic role of oxidised LDL in coronary artery disease, it is plausible that these demonstrable reductions in oxLDL and their associated antibodies (or immune complexes) may represent one of the mechanisms by which LA leads to clinical benefits in patients with refractory angina and raised $\mathrm{Lp}(\mathrm{a})$.

\section{SIGNIFICANT MUTUAL SERVICE BENEFITS FROM A REGULAR ANAESTHETIC PRE-ASSESSMENT CARDIOLOGY MULTIDISCIPLINARY TEAM MEETING}

${ }^{1}$ Hannah Cranch*, ${ }^{2}$ Adam James, ${ }^{2}$ Sophie Laffan, ${ }^{2}$ Peter Fitzgerald, Robert.A Bleasdale 2. ${ }^{1}$ Hwyel Dda University Health Board; ${ }^{2}$ Cwm Taf University Health Board

\subsection{6/heartjnl-2017-311726.94}

Introduction Cardiovascular complications are one of the major causes of mortality and morbidity perioperatively. Anaesthetic assessment involving a cardiologist reduces perioperative cardiovascular complications; however assessment in the outpatient setting can incur significant waiting times. A weekly cardiology, anaesthetic and pre-assessment multidisciplinary team (MDT) meeting was introduced with the aim of reducing the time taken to optimise patients for anaesthesia and reduce the burden on the cardiology outpatient waiting list.

Methods A weekly MDT was commenced in October 2015, discussing patients who posed a cardiovascular concern to the pre-assessment unit. Outcomes measured included time taken to be optimised for anaesthesia, need for cardiology outpatient review and investigations required. A comparison was made between those seen in pre-assessment clinic, prior to the initiation of the MDT (retrospective) and those discussed in MDT (prospective). A six month period, October-April, one year apart was studied. The data was collected from an electronic Pre-assessment system designed in our institution.

Results In the prospective period, 80 patients were discussed, with an average of $10 \mathrm{~min}$ being spent discussing each patient. There was a $10 \%$ reduction in number of investigation performed. The number of patients requiring formal cardiology outpatient review was significantly reduced in the prospective group (10/80) compared to the retrospective group (50/50) $(p=0.0001)$. The time taken to optimise patients for anaesthesia was reduced by 76 days $(p=0.0002)$ by introducing the MDT. This $49 \%$ reduction was achieved without an increase in cardiac testing.

Abstract 95 Table 1

\begin{tabular}{lll}
\hline & Retrospective & Prospective \\
\hline Number sent for Cardiology outpatient review & $50 / 50$ & $10 / 80$ \\
Average time to optimisation (days) & 155 & 79 \\
Percentage of patients sent for further investigation & 54 & 44 \\
\hline
\end{tabular}

